\title{
Subfactors and Paragroup Theory
}

\author{
YASUYUKI KAWAHIGASHI \\ Department of Mathematical Sciences \\ University of Tokyo, Komaba, Tokyo, 153, JAPAN
}

\begin{abstract}
We survey the current status of Ocneanu's paragroup theory and its relation to topological quantum field theory.
\end{abstract}

\section{Introduction}

Since V. F. R. Jones initiated the subfactor theory in [16], we have seen many new combinatorial structures arising from subfactors. It is Ocneanu's paragroup theory [23] that describes the combinatorial structures and relates the subfactor theory to other new fields such as the quantum group theory, rational conformal field theory, topological quantum field theory, etc. We see a great deal of similarity among the combinatorial structures of these theories, but we also have subtle differences. We now survey the current status of the paragroup theory and its relations to the other topics.

\section{General theory of paragroups}

We start with a subfactor $N \subset M$ of type $\mathrm{II}_{1}$ with finite Jones index. (In this paper, we always assume these conditions when we say a "subfactor" $N \subset M$.) The basic construction of Jones [16] produces the increasing sequence of $\mathrm{II}_{1}$ factors, the Jones tower:

$$
N \subset M \subset M_{1} \subset M_{2} \subset M_{3} \subset \cdots
$$

We have a double sequence of finite dimensional algebras $\left\{M_{k}^{\prime} \cap M_{l}\right\}_{-1 \leq k \leq l}$ called the higher relative commutants as an invariant of the subfactor, where we have set $M_{0}=M$ and $M_{-1}=N$.

This double sequence is known to be a complete invariant for a subfactor of the hyperfinite $\mathrm{II}_{1}$ factor with finite index and strong amenability by a deep theorem of Popa [33]. A paragroup is an algebraic system introduced by Ocneanu [23] to characterize this double sequence in a combinatorial way, when we assume the finite depth condition for a subfactor. (We will give the definition for the finite depth condition later. It is a stronger condition than the strong amenability.) A finite 
group gives an example of a paragroup, and a general paragroup is regarded as a generalization, or a quantization, of a notion of a finite group. (This quantization is different from that for quantum groups. See the next Section.)

Ocneanu has two equivalent axiomatizations of paragroups as in [23], [25]. We explain the approach in [25] as follows. (See [11, Chapters 9-12] for details of the both approaches.)

From a subfactor $N \subset M$ of type $\mathrm{II}_{1}$ with finite index, we get a bimodule ${ }_{N} L^{2}(M)_{M}$. Using the relative tensor product of Connes, we get finite tensor powers

$$
\cdots \otimes_{N} L^{2}(M) \otimes_{M} L^{2}(M) \otimes_{N} L^{2}(M) \otimes_{M} \cdots
$$

and make irreducible decompositions. We get four kinds of bimodules; $N-N, N-M$, $M-N$, and $M-M$. The finite depth assumption means that we get only finitely many isomorphism classes of bimodules in this procedure. In this Section, we assume that $M$ is hyperfinite and $N \subset M$ is of finite depth. If we make a relative tensor product of two bimodules arising in this procedure, we get a direct sum of bimodules also arising in the same way. We call such a set of (isomorphism classes of) bimodules a system of bimodules arising from the subfactor. The rule of irreducible decompositions of relative tensor products of two bimodules is called a fusion rule of the system. This is an analogue of the decomposition rule of tensor products of two representations of a compact group. A bimodule map is called an intertwiner as an analogue of an intertwiner of representations. Associativity of the relative tensor product gives two ways of representing $X \otimes Y \otimes Z$ and the compatibility of the two ways produces so-called quantum $6 j$-symbols by composing intertwiners, as a direct analogue of $6 j$-symbols for representations of a compact group. The fusion rule and quantum $6 j$ symbols completely determine the system of bimodules and by axiomatizing them, we get a paragroup. In this way, a paragroup is a combination of a fusion rule (of four kinds of bimodules) and an equivalence class of quantum $6 j$-symbols on it. (See [11, Chapter 12] for exact definitions of quantum $6 j$-symbols, their equivalence relation, etc.) That is, in an abstract formulation, we have a finite set of "irreducible" objects and we have an operation called a "tensor product". We also have an irreducible decomposition of a tensor product of two objects. We have intertwiners between objects and require that self-intertwiners on an irreducible object give a complex number. Then we can define $6 j$-symbols and we require them to satisfy the axioms called unitarity, tetrahedral symmetry, and pentagonal relations. In this way, we have a categorical axiomatization and such a system is sometimes called a fusion category. Two important points of paragroups in this categorical formulation are that the "tensor product" is not commutative in general and that each object has a positive "dimension".

For a subfactor $N \subset M$, we consider the two graphs defined as follows. We label the vertices with (the isomorphism classes of) the four kinds of bimodules arising from the subfactor as above. We connect an $N-N$ bimodule $X$ and an $N-M$ bimodule $Y$ with edges of multiplicity $\operatorname{dim} \operatorname{Hom}\left({ }_{N} X \otimes_{N} L^{2}(M)_{M}, Y\right)$, where Hom means the space 
of the $N-M$ bimodule maps. We also connect an $M-M$ bimodule $X$ and an $M-N$ bimodule $Y$ with edges of multiplicity $\operatorname{dim} \operatorname{Hom}\left({ }_{M} X \otimes_{M} L^{2}(M)_{N}, Y\right)$. on this way, we get two graphs; one has vertices labelled with $N-N$ bimodules and $N-M$ bimodules and the other with $M-M$ bimodules and $M-N$ bimodules. We call these graphs the principal graph and the dual principal graph of the subfactor $N \subset M$ respectively. These two graphs give only partial data of the paragroup of $N \subset M$, but in many concrete cases these graphs determine the paragroup (almost) uniquely, so we often draw these graphs to represent a paragroup.

When the Jones index is less than or equal to four, the (dual) principal graphs must be one of the (extended) Dynkin diagrams of type $A, D$, or $E$. A complete classification of the corresponding paragroups has been given by Ocneanu, Popa, and various people. (See [11, Chapter 11]. Consideration of fusion rules is quite useful as in [15].)

Ocneanu has recently proved the following theorem on a general structure of paragroups and presented his proof in [30].

Theorem 2.1 For a given fusion rule, we have only finitely many equivalence classes of quantum $6 j$-symbols.

For a fixed fusion rule, quantum $6 j$-symbols are just finite sets of linear maps on finite dimensional Hilbert spaces satisfying some axioms. It is easy to see that the set of such linear maps is compact. Ocneanu works on this compact space to prove the above theorem. The following is Ocneanu's corollary to the above theorem. (See [19] for more explanations.)

Corollary 2.2 For any finite graph, we have only finitely many paragroups having this graph as a principal graph.

\section{Known examples}

In this section, we survey known constructions of paragroups/subfactors. First we list constructions giving "basic" examples and then describe methods to construct new examples from given ones.

The following three tools have been used for constructing subfactors of finite index and finite depth.

- Finite groups/finite dimensional Hopf $C^{*}$-algebras

- Quantum groups and rational conformal field theory

- Exceptional paragroups

We also have a method of construction using another mathematical object such as loop groups as in [46], [54], [55] but it gives the same paragroups as the ones arising from the second in the above list, so we have not listed such a construction separately. 
The first case just means a subfactor given as $R \subset R \times H$, where $H$ is a finite group or a finite dimensional Hopf $C^{*}$-algebra acting on $R$ freely. (Note that we always have such an action on the hyperfinite $\mathrm{II}_{1}$ factor for any finite group or a finite dimensional Hopf $C^{*}$-algebra.) This construction has been known for a long time, and there is nothing mysterious in it today.

The construction of subfactors from quantum groups has been studied extensively by Wenzl [47], [48], [49]. These subfactors arise from quantum groups at roots of unity of the form $q=\exp \pi i / n$. From the viewpoint of the paragroup theory based on quantum $6 j$-symbols, the easiest way to understand such a construction is a categorical method. As noticed in [6], we can get a paragroup from another categorical structure arising a from Wess-Zumino-Witten model or a quantum group. (Also see [44]. Seeing this kind of construction, Ocneanu has noticed the above-mentioned equivalence of the two axiomatizations of paragroups.)

We can extend this construction to the orbifold subfactors in [8], [18], [51], the Goodman-de la Harpe-Jones subfactors and their generalizations in [12], [52], and subfactors corresponding to the Dynkin diagrams $E_{6}, E_{8}$ (see [53]) by working on finer mathematical structures. For this purpose, it is better to use rational conformal field theory rather than quantum groups. See [11, Chapter 13]. (From our combinatorial viewpoints, these two mathematical structures are quite similar, though they are originally different.)

From today's viewpoint, the above two constructions are not very surprising any more in the sense that these constructions do not produce previously unknown mathematical structures. So it would be desirable to find subfactors not arising from such constructions. Haagerup has tried to find such subfactors.

The above mentioned classification of subfactors with index less than or equal to four has been done by combinatorial arguments. Haagerup further made this kind of combinatorial studies in detail and has obtained a list of candidates of (dual) principal graphs for subfactors with index in the interval $(4,3+\sqrt{3}]$ that might be realized from subfactors in [13]. His list has the graph $A_{\infty}$ as a "generic case" and two series of pairs of finite graphs and one pair of finite graphs. It was not clear at all which graphs of his list are indeed realized as a principal graph of a subfactor, except for the pair of finite graphs for the index value $(5+\sqrt{13}) / 2$ for which he announced that it is indeed realized from a subfactor of the hyperfinite $\mathrm{II}_{1}$ factor. This is the smallest possible index value above four for subfactors with finite depth. This pair of the principal and dual principal graphs is given as in Fig. 1. The two copies of the principal graph and the two copies of the dual principal graphs are connected as in Fig. 2, where the top four vertices are naturally identified with the bottom four vertices. This diagram gives the Bratteli diagram of the canonical commuting square of the subfactor in the sense of Popa [31]. (Haagerup's proof of the realization has been recently written in [1].)

The realization of the other graphs from a subfactor had been an open problem for some years, but we recently see some progress on this problem. Bisch has proved 

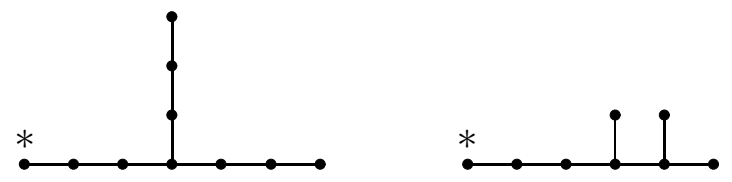

Figure 1. The (dual) principal graphs of the Haagerup subfactor

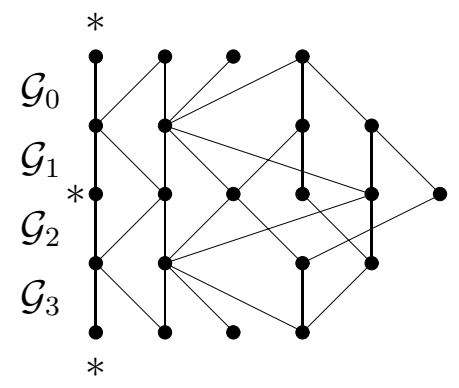

Figure 2.

that one series of the graphs is not realized at all in [3] by showing inconsistency of the fusion rules. Asaeda and Haagerup have proved in [1] that one exceptional pair of the graphs in the Haagerup list is indeed realized. The graphs are listed in Fig. 3.

An outline of their method of the realization is as follows. Assuming that the graphs are indeed the (dual) principal of a subfactor, we get a partial information on the fusion rule. This partial information often (but not always) determines the entire fusion rule of the subfactor, and this is the case for the Haagerup and AsaedaHaagerup subfactors. Then by reversing the computations, we can often prove that a few identities for the fusion rules conversely determine the (dual) principal graphs as desired. In the case of the Haagerup and Asaeda-Haagerup subfactors, it turns out
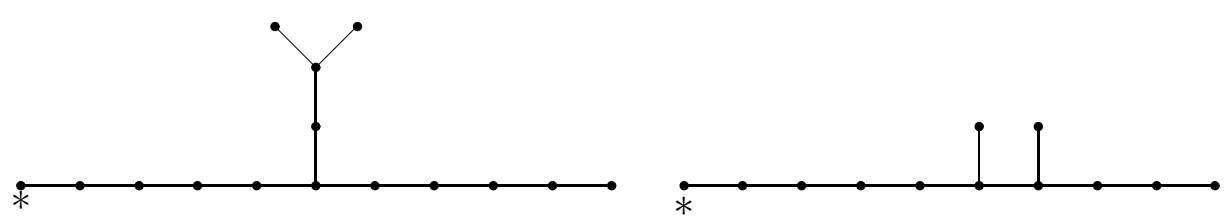

Figure 3. The (dual) principal graphs of the Asaeda-Haagerup subfactor 
that verifying one identity for the fusion rules is enough in each case. This verification involves relative tensor products of bimodules, that is, infinite dimensional Hilbert spaces. Asaeda and Haagerup [1] have established a method to reduce this infinite dimensional problem to computations of a finite number of finite dimensional unitary matrices. This is the method of open string bimodules which generalizes Ocneanu's construction [23] and Sato's generalization [39]. (This is similar to Ocneanu's work in [29], but there is a subtle difference for the equivalence relation of connections in terms of gauge choices.) Even after this reduction, the computations are highly complicated, but they managed to complete the computations as in [1]. This method of open string bimodules will be very useful in the future studies of subfactors.

At present, these two examples of Haagerup and Asaeda-Haagerup are the only known genuine exceptional subfactors that do not arise from finite or quantum groups. Note that these have no deformation parameter $q$. (We have no unified definition of quantum groups. Here we mean by a "quantum group" a certain Hopf algebra arising as a deformation of a Lie algebra.)

In the original axiomatization of a paragroup, this kind of realization problem is reduced to numerical identities. In Ocneanu's terminology, this is a flatness problem of a bi-unitary connection. (See [11, Chapter 11] for a general theory.) These numerical identities are extremely complicated and hard to verify, but in some cases approximate computations on a computer is possible. Ikeda has made such computations and predicted the existence of the above-mentioned Asaeda-Haagerup subfactor in [14]. He further made an approximate verification of the flatness for the second pair of the graphs of the last series in the Haagerup list, as in Fig. 4, and found that the identities are "almost" valid. (The first pair of the graphs of this last series is for the Haagerup subfactor of index $(5+\sqrt{13}) / 2$ as in Fig. 1.) We, however, still do not have a rigorous proof of the realization of these graphs in Fig. 4. Since the first pair of graphs of the series is realized and the second is very "likely" to be realized, we expect that all of the series are indeed realized, but no concrete evidence has been obtained. This series will be very interesting from the viewpoint of topological invariants in three-dimension which we will explain in the last section.

From these examples, we expect that we would have more and more exotic subfactors not arising from quantum groups, if we go beyond the above index range $(4,3+\sqrt{3})$, but the combinatorial problems become exponentially difficult, and no concrete results have been known.

Next, we have the following method to produce new subfactors from given ones.

- Tensor product

- Intermediate subfactor

- Composition

- Asymptotic inclusion 


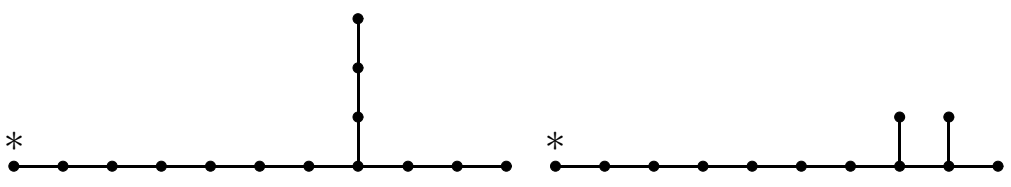

Figure 4. A candidate of the (dual) principal graphs by Haagerup

The tensor product construction produces $N \otimes P \subset M \otimes Q$ from two subfactors $N \subset M$ and $P \subset Q$. The Jones indices are multiplied and we can say this construction gives a direct product of two paragroups. The resulting subfactor is of finite depth if and only if so are the both subfactors. Nothing is mysterious in this construction.

The next construction produces $N \subset R, R \subset M$ from a subfactor $N \subset M$. Bisch [2] has shown that if we have a finite depth for $N \subset M$, then we automatically have a finite depth for $N \subset R, R \subset M$. A criterion for existence of such an $R$ has been given in [2], [24]. If we start with a subfactor $N \subset N \times G=M$, where $G$ is a finite group acting on $N$ freely, then all the intermediate factors $R$ are of the form $N \times H$ where $H$ is a subgroup of $G$. Then as intermediate subfactors, we get $N \subset N \times H$ and $N \times H \subset N \times G$. If we identify the paragroup of the subfactor $N \subset N \times G$ with the group $G$, these two subfactors give paragroups corresponding to the subgroup $H$ and the quotient $G / H$. For a general subfactor $N \subset M$, we have no reason to distinguish the classes of $N \subset R$ and $R \subset M$, which means, roughly speaking, we have no distinction of a subsystem and a quotient for paragroups.

The composition of subfactors is a converse construction to the above. That is, from $N \subset M$ and $M \subset R$, we get $N \subset R$. Note that even if we fix the isomorphism classes of $N \subset M$ and $M \subset R$, that of $N \subset R$ is not uniquely determined. By controlling the relation of the original two subfactors, one can construct some subtle examples as in [4]. In a "generic" case, even when $N \subset M$ and $M \subset R$ are of finite depth, we would get an infinite depth for $N \subset R$. In such a case, the paragroup of $N \subset R$ is a free product of the two paragroups of $N \subset M$ and $M \subset R$ in some sense as in $[5]$.

The last one, the asymptotic inclusion, has been introduced by Ocneanu [23], [24]. From a subfactor $N \subset M$ of the hyperfinite $\mathrm{II}_{1}$ factor with finite index and finite depth, this construction produces a new subfactor $M \vee\left(M^{\prime} \cap M_{\infty}\right) \subset M_{\infty}$, where $M_{\infty}$ is the weak clusre of the union of the Jones tower with respect to the trace. This new inclusion has finite index if and only if the original inclusion is of finite depth. This construction is related to topological quantum field theory and it gives a paragroup analogue of the quantum double construction of Drinfel'd [7]. See [10], [11, Chapters 12-13], [21], [22] for more on asymptotic inclusions.

The paragroup of the asymptotic inclusion is dual to that of the central sequence 


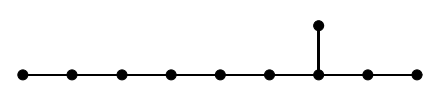

Figure 5 .

subfactor $N^{\omega} \cap M^{\prime} \subset M_{\omega}$, where $\omega$ is a free ultrafilter over the positive integers. (We say that the paragroup of $M \subset M_{1}$ is dual to that of $N \subset M$.) See [11, Chapter 15], [20] for more on central sequence subfactors.

\section{Infinite depth case}

In this section, we discuss what is known for subfactors of infinite depth. S. Popa [33] has proved that if a subfactor is strongly amenable, then it is "recovered" from its higher relative commutants. He has obtained various characterization of strong amenability in [33] (also see [35], [36]) and the abstract theory for strong amenability has made much advance due to his series of work. We, however, do not have many "natural" examples of strongly amenable subfactors without finite depth, except for those arising from group actions such as "locally trivial subfactors" or those in [4], [45].

As mentioned above, Haagerup [13] has shown a list of candidates of (dual) principal graphs for the index range $(4,3+\sqrt{3}]$ by complicated and subtle combinatorial arguments. His work, in particular, implies that if the index value is between 4 and $(5+\sqrt{13}) / 2=4.302 \cdots$, then the principal graph must be $A_{\infty}$. Haagerup-Schou [41] and Ocneanu have constructed many subfactors of the hyperfinite $\mathrm{II}_{1}$ factors with trivial relative commutants with indices in this range using bi-unitary connections on finite graphs. In this way, the Jones index we get is the square of the PerronFrobenius eigenvalue of the graphs we use. Then a result in the graph theory implies that the smallest index value above four we can construct in this way is $4.026 \cdots$, even if we allow infinite graphs. (See also Appendix I of [12].) This value is the square

of the Perron-Frobenius eigenvalue of the graph in Fig. 5. Ocneanu's construction of a subfactor with trivial relative commutant for this index value based on the graph in Fig. 5 is described in [41]. By the above remark, this subfactor has $A_{\infty}$ as (dual) principal graphs.

Popa has also proved that any value bigger than 4 can be realized as a Jones index of some subfactor with trivial relative commutant of a $\mathrm{II}_{1}$ factor which is not hyperfinite in [32]. Thus, if we drop the hyperfiniteness, we have a complete answer of possible values of the Jones index of general subfactors with trivial relative commutant, but the problem of the range of the possible Jones index values of subfactors of the hyperfinite $\mathrm{II}_{1}$ factor with trivial relative commutant is still open. 


\section{Relation to topological quantum field theory}

It was the discovery of the Jones polynomial [17] that has connected the theory of operator algebras to low dimensional topology. Witten [50] has formulated 3dimensional topological quantum field theory, in the sense of Atiyah, based on the Jones polynomial.

There have been several methods to construct 3-dimensional topological quantum field theory rigorously from a set of combinatorial data, but from the viewpoint of the paragroup theory, the following two are the most important. One is the construction of Turaev-Viro [43] using triangulation and the other is the one of Reshetikhin-Turaev [37] using surgery. (In these constructions, we regard a compact 3-manifold is made of a finite number of tetrahedra or with a Dehn surgery on a link. We define a complex number for a closed manifold using the triangulation or a link and then prove that the number does not depend on a particular triangulation or a link. See [42] for a general theory in detail.)

It was Ocneanu who realized these constructions can be applied with combinatorial data arising from a paragroup. That is, we can use the quantum $6 j$-symbols arising from subfactors with finite depth for the Turaev-Viro method and the braiding arising from the asymptotic inclusion of a subfactor with finite depth for the Reshetikhin-Turaev method. Ocneanu has presented his theory in [25], [26], [27], [28]. (Also see [9], [10] [11, Chapter 12].) It seems that the Turaev-Viro type topological quantum field theory arising from the quantum $6 j$-symbols of $N \subset M$ is identical to the Reshetikhin-Turaev type topological quantum field theory arising from the natural braiding on the asymptotic inclusion $M \vee\left(M^{\prime} \cap M_{\infty}\right) \subset M_{\infty}$, but this has not been proved yet.

It is also an interesting problem to define a topological quantum field theory from subfactors with infinite depth. Amenability is expected to play an important role here.

Another important notion connected to topological quantum field theory is equivalence of systems of bimodules as defined in [25]. We say that the systems of $N-N$ bimodules and those of $M-M$ bimodules arising from a subfactor $N \subset M$ with finite depth are equivalent. Based on this, Sato [40] has defined an equivalence for subfactors and studied its relation to commuting squares in [38], [39], [40]. Ocneanu [25] has noticed that equivalent subfactors give the same Turaev-Viro type topological quantum field theory. (See [9], [11, Chapter 12].) It is an interesting open problem to determine whether two subfactors producing the same Turaev-Viro type topological quantum field theory are equivalent or not. We expect that the answer would be affirmative.

\section{References}

[1] M. Asaeda \& U. Haagerup, Exotic subfactors of finite depth with Jones index 
$(5+\sqrt{13}) / 2$ and $(5+\sqrt{17}) / 2$, preprint 1998.

[2] D. Bisch, A note on intermediate subfactors, Pac. J. Math. 163 (1994), 201-216.

[3] D. Bisch, Principal graphs of subfactors with small Jones index, to appear in Math. Ann.

[4] D. Bisch \& U. Haagerup, Composition of subfactors: New examples of infinite depth subfactors, Ann. Sci. École Norm. Sup. 29 (1996), 329-383.

[5] D. Bisch \& V. F. R. Jones. Algebras associated to intermediate subfactors, Invent. Math. 128 (1997), 89-157.

[6] J. de Boer \& J. Goeree, Markov traces and $I I_{1}$ factors in conformal field theory, Comm. Math. Phys. 139 (1991), 267-304.

[7] V. G. Drinfel'd, Quantum groups, Proc. ICM-86, Berkeley, 798-820.

[8] D. E. Evans \& Y. Kawahigashi, Orbifold subfactors from Hecke algebras, Comm. Math. Phys. 165 (1994), 445-484

[9] D. E. Evans \& Y. Kawahigashi, From subfactors to 3-dimensional topological quantum field theories and back - a detailed account of Ocneanu's theory -, Internat. J. Math. 6 (1995), 537-558.

[10] D. E. Evans \& Y. Kawahigashi, On Ocneanu's theory of asymptotic inclusions for subfactors, topological quantum field theories and quantum doubles, Internat. J. Math. 6 (1995), 205-228.

[11] D. E. Evans \& Y. Kawahigashi, "Quantum symmetries on operator algebras", Oxford University Press, 1998.

[12] F. Goodman, P. de la Harpe, \& V. F. R. Jones, "Coxeter graphs and towers of algebras", MSRI publications 14, Springer, 1989.

[13] U. Haagerup, Principal graphs of subfactors in the index range $4<[M: N]<$ $3+\sqrt{2}$, in "Subfactors - Proceedings of the Taniguchi Symposium, Katata", (ed. H. Araki et al.), World Scientific, Singapore, pp. 1-38.

[14] K. Ikeda, Numerical evidence for flatness of Haagerup's connections, preprint 1997.

[15] M. Izumi, Application of fusion rules to classification of subfactors, Publ. RIMS Kyoto Univ. 27 (1991), 953-994.

[16] V. F. R. Jones, Index for subfactors, Invent. Math. 72 (1983), 1-15. 
[17] V. F. R. Jones, A polynomial invariant for knots via von Neumann algebras, Bull. Amer. Math. Soc. 12 (1985), 103-112.

[18] Y. Kawahigashi, On flatness of Ocneanu's connections on the Dynkin diagrams and classification of subfactors, J. Funct. Anal. 127 (1995), 63-107.

[19] Y. Kawahigashi, Quantum doubles and orbifold subfactors, in "Operator Algebras and Quantum Field Theory", (ed. S. Doplicher et al) (1997),3 271-283, International Press.

[20] K. Kawamuro, Central sequence subfactors and double commutant properties, preprint 1998.

[21] R. Longo \& K.-H. Rehren, Nets for subfactors, Rev. Math. Phys. 7 (1995), 567597.

[22] T. Masuda, An analogue of Longo's canonical endomorphism for bimodule theory and its application to asymptotic inclusions, to appear in Internat. J. Math. 8 (1997), 249-265.

[23] A. Ocneanu, Quantized group string algebras and Galois theory for algebras, in "Operator algebras and applications, Vol. 2 (Warwick, 1987)," London Math. Soc. Lect. Note Series Vol. 136, Cambridge University Press, 1988, pp. 119-172.

[24] A. Ocneanu, "Quantum symmetry, differential geometry of finite graphs and classification of subfactors", University of Tokyo Seminary Notes 45, (Notes recorded by Y. Kawahigashi), 1991.

[25] A. Ocneanu, An invariant coupling between 3-manifolds and subfactors, with connections to topological and conformal quantum field theory, preprint 1991.

[26] A. Ocneanu, Lectures at Collège de France, Fall 1991.

[27] A. Ocneanu, Seminar talk at University of California, Berkeley, June 1993.

[28] A. Ocneanu, Chirality for operator algebras, (recorded by Y. Kawahigashi), to appear in "Subfactors - Proceedings of the Taniguchi Symposium, Katata, 1993".

[29] A. Ocneanu, Paths on Coxeter diagrams: From Platonic solids and singularities to minimal models and subfactors, in preparation.

[30] A. Ocneanu, Conference talk at Madras, India, January 1997.

[31] S. Popa, Classification of subfactors: reduction to commuting squares, Invent. Math. 101 (1990), 19-43. 
[32] S. Popa, Markov traces on universal Jones algebras and subfactors of finite index, Invent. Math. 111 (1993), 375-405.

[33] S. Popa, Classification of amenable subfactors of type II, Acta Math. 172 (1994), $253-345$.

[34] S. Popa, An axiomatization of the lattice of higher relative commutants of a subfactor, Invent. Math. 120 (1995), 427-446.

[35] S. Popa, On Connes' joint distribution trick, to appear in Ens. Math.

[36] S. Popa, Some properties of the symmetric enveloping algebra of a subfactor, with applications to amenability and property T, preprint 1998.

[37] N. Yu. Reshetikhin \& V. G. Turaev, Invariants of 3-manifolds via link polynomials and quantum groups, Invent. Math. 103 (1991), 547-597.

[38] N. Sato, Two subfactors arising from a non-degenerate commuting square - An answer to a question raised by V. F. R. Jones-, Pac. J. Math. 180 (1997), 369-376.

[39] N. Sato, Two subfactors arising from a non-degenerate commuting square Tensor categories and TQFT's-, Internat. J. Math. 8 (1997), 407-420.

[40] N. Sato, Constructing a non-degenerate commuting square from equivalent systems of bimodules, to appear in Internat. Math. Research Notices.

[41] J. Schou, Commuting squares and index for subfactors, Ph. D. Thesis, Odense University, 1990.

[42] V. G. Turaev, "Quantum invariants of knots and 3-manifolds", de Gruyter Studies in Mathematics, Vol. 18, (1994).

[43] V. G. Turaev \& O. Y. Viro, State sum invariants of 3-manifolds and quantum 6j-symbols, Topology 31 (1992), 865-902.

[44] V. G. Turaev \& H. Wenzl, Quantum invariants of 3-manifolds associated with classical simple Lie algebras, Internat. J. Math. 4 (1993), 323-358.

[45] A. J. Wassermann, Coactions and Yang-Baxter equations for ergodic actions and subfactors, in "Operator algebras and applications", (eds. D. E. Evans and M. Takesaki), London Mathematical Society Lecture Notes, 136 (1988), 203-236.

[46] A. J. Wassermann, Operator algebras and conformal field theory III: Fusion of positive energy representations of $S U(N)$ using bounded operators, to appear in Invent. Math. 
[47] H. Wenzl, Hecke algebras of type A and subfactors, Invent. Math. 92 (1988), $345-383$.

[48] H. Wenzl, Quantum groups and subfactors of type B, C, and D, Comm. Math. Phys. 133 (1990), 383-432.

[49] H. Wenzl, $C^{*}$-tensor categories from quantum groups, preprint 1997.

[50] E. Witten, Quantum field theory and Jones polynomial, Comm. Math. Phys. 121 (1989), 351-399.

[51] F. Xu, Orbifold construction in subfactors, Comm. Math. Phys. 166 (1994), $237-254$.

[52] F. Xu, Generalized Goodman-Harpe-Jones construction of subfactors I, II, Comm. Math. Phys. 166 (1997), 475-491, 493-508.

[53] F. Xu, New braided endomorphisms from conformal inclusions, to appear in Comm. Math. Phys.

[54] F. Xu, Jones-Wassermann subfactors for disconnected intervals, preprint 1997.

[55] F. Xu, Applications of braided endomorphisms from conformal inclusions, to appear in Internat. Math. Research Notices. 